Proceedings

\title{
Comparison of Microsatellites and SNP Markers in Genetic Diversity Level of Two Scots Pine Stands ${ }^{\dagger}$
}

\author{
Anna Tereba ${ }^{1 *}$ and Agata Konecka ${ }^{2}$ \\ 1 Department of Forest Ecology, Forest Research Institute, 3 Braci Leśnej St., 05-090 Sękocin Stary ; \\ A.Tereba@ibles.waw.pl \\ 2 Faculty of Forestry, Agriculture University, ul. Nowoursynowska 159, 02-776 Warszawa; agataludw@o2.pl \\ * Correspondence: A.Tereba@ibles.waw.pl \\ + Presented at the 1st International Electronic Conference on Forests, 15-30 November 2020; \\ Available online: https://sciforum.net/conference/IECF2020 \\ Published: 25 October 2020
}

\begin{abstract}
Scots pine (Pinus silvestris), is one of the dominant species in Poland and one of the main forest tree species in northern and central Europe. This species is of great economic importance. The Scots pine is highly adaptable to changing environmental conditions. A number of ecotypes have been characterized and formation of these ecotypes are related with development of different phenotypic characteristics: morphological, physiological and ecological. Molecular studies, based on DNA polymorphism, have been used for more than 20 years to analyzed genetic diversity of Scots pine population. The most popular are microsatellite markers due to the fact of wide availability and high polymorphism. However, the use of these markers is also associated with certain limitations: due to complex mutation models or high incidence of homoplasia. These features are prompting scientists to look for alternative types of markers such as for example SNP. In our study we conducted a comparison of the basic parameters of genetic variability of two Scots pine stands ( 25 and 24 trees in each) for 20 SNP markers and 4 microsatellite markers. For the 20 SNP loci the observed heterozygosity (Ho) was equal 0,34 for both stands and the expected heterozygosity (He) for the first stand was equal 0,34 and for the second 0,37 . No statistical significant genetic distance was observed between them. For the microsatellite markers observed heterozygosity (Ho) was 0,81 and 0,74 and the expected heterozygosity $(\mathrm{He}$ ) was equal 0,85 and 0,85 respectively for stands and similarly no statistical significant genetic distance was observed.
\end{abstract}

Literature data of different genetic markers showed higher informativeness of random chosen microsatellite than SNP markers for study population differentiation. But some analyzes confirm that the appropriate number of SNP markers can be more informative for population structure inference.

Keywords: Pinus silvestris; molecular markers; genetic diversity

\section{Introduction}

Knowledge about the history of the population and the relationships between individuals in populations is extremely important for many studies in the field of genetics, molecular biology and conservation genetics. For many years, there has been great interest in the scientific community in the use of genetic information to infer about various population parameters. Genetic markers is widely used, e.g. for the estimation of relationship [1], [2], the inbreeding coefficient [3] or the intensification of migration both in the global and local perspective [2]. Such studies are often limited to microsatellite marker panels, but in some cases there are not enough loci or alleles to reliably infer the desired parameters. For a large number of scientific studies, microsatellite markers are molecular markers of the "first choice" due to the fact of wide availability and high polymorphism. However, the use of these markers is also associated with certain limitations: due to complex mutation models 
[4]; high incidence of homoplasia [5]; error frequency and low genotypic throughput [6]. These features are prompting scientists to look for alternative types of markers. As the amount of data in genomics increases, the availability of single nucleotide polymorphisms (SNPs) continues to increase, also for non-model organisms, contributing to the growing interest of these markers in the field of population genetics. SNP polymorphisms are characterized by high variance in the genome for most organisms and, importantly, they are distributed throughout the genome with high frequency.

\section{Materials and Methods}

DNA was extracted using the NucleoSpin ${ }^{\circledR}$ Plant II (Machery Nagel). The amplification of microsatellite DNA fragments was carried out by polymerase chain reaction (PCR), using the Qiagen ${ }^{\circledR}$ Multiplex PCR Kit. Analysis of nuclear microsatellite sequences (nSSR) were performed according to a modified procedure by [7] using three microsatellite loci: SPAG 7.14, SPAC 11.6 and SPAC 12.5 and according to [8] for the SsrPt_ctg4363 locus.

SNP sites were selected, accordingly with maximum numbers of SNP's in genes and the possibility of designing primers for multiplex reaction. Primers were designed in the Primer 3 program [9], [10] as predicted for the SNP analysis by primer extension and nucleotide termination (single-based extension and termination) [11] method with ABI Prism SNaPshot Multiplex Kit. The SNP was identified by SNP genotyping and confirmed by sequencing. Basic genetic parameters were calculated in GenAlEx 6.5 [12] and Arlequin 3.5 [13].

\section{Results}

The results of genetic differentiation obtained on the basis of the analysis of 4 microsatellite markers showed a high level of both observed and expected heterozygosity in both stands for all analyzed loci (Tab. 1). Mean value of observed heterozygosity (Ho) in both stands was 0,81 and 0,74 and the expected heterozygosity $(\mathrm{He})$ was equal 0,85 and 0,85 respectively. Clearly lower values of these coefficients were obtained in the case of the analysis of the polymorphism of 20 single nucleotides (Tab. 1). No statistical significant genetic distance between stands was observed alike in the case of SSR markers and SNP markers.

Table 1. Mean values of observed heterozygosity (Ho) and expected heterozygosity $(\mathrm{He})$ for both type of molecular markers.

\begin{tabular}{ccccc}
\hline Stand & Ho SSR & He SSR & Ho SNP & HE SNP \\
\hline Pop 1 & 0,81 & 0,85 & 0,34 & 0,34 \\
\hline Pop 2 & 0,74 & 0,85 & 0,34 & 0,37 \\
\hline
\end{tabular}

\section{Discussion}

Literature data of different genetic markers showed higher informativeness of random chosen microsatellite than SNP markers for study population differentiation [14]. But some analyzes confirm that the appropriate number of SNP markers can be more informative for population structure inference [15]. In the studies of the genetic variability of Scots pine a microsatellite markers are the basic tools, but the SNP markers also carried out in the context of analyzing the degree of polymorphism and the ability to adapt in a changing environment.

\section{References}

1. Queller, D.C.; Goodnight, K.F. ESTIMATING RELATEDNESS USING GENETIC MARKERS. Evolution (N. Y). 1989, 43, 258-275, doi:doi:10.1111/j.1558-5646.1989.tb04226.x.

2. Ritland, K. A MARKER-BASED METHOD FOR INFERENCES ABOUT QUANTITATIVE INHERITANCE IN NATURAL POPULATIONS. Evolution (N. Y). 1996, 50, 1062-1073, doi:10.1111/j.1558-5646.1996.tb02347.x. 
3. Lynch, M.; Ritland, K. Estimation of Pairwise Relatedness With Molecular Markers. Genetics 1999, 152, 1753-1766.

4. Di Rienzo, A.; Peterson, A.C.; Garza, J.C.; Valdes, A.M.; Slatkin, M.; Freimer, N.B. Mutational processes of simple-sequence repeat loci in human populations. Proc. Natl. Acad. Sci. 1994, 91, 3166 LP - 3170, doi:10.1073/pnas.91.8.3166.

5. Estoup, A.; Garnery, L.; Solignac, M.; Cornuet, J.M. Microsatellite variation in honey bee (Apis mellifera L.) populations: hierarchical genetic structure and test of the infinite allele and stepwise mutation models. Genetics 1995, 140, 679-695.

6. Hoffman, J.I.; Amos, W. Microsatellite genotyping errors: detection approaches, common sources and consequences for paternal exclusion. Mol. Ecol. 2005, 14, 599-612, doi:10.1111/j.1365-294X.2004.02419.x.

7. Soranzo, N.; Provan, J.; Powell, W. Characterization of microsatellite loci in Pinus sylvestris L. Mol. Ecol. $1998,7,1260-1261$.

8. Chagné, D.; Chaumeil, P.; Ramboer, A.; Collada, C.; Guevara, A.; Cervera, M.T.; Vendramin, G.G.; Garcia, V.; Frigerio, J.-M.; Echt, C.; et al. Cross-species transferability and mapping of genomic and cDNA SSRs in pines. Theor. Appl. Genet. 2004, 109, 1204-1214, doi:10.1007/s00122-004-1683-z.

9. Untergasser, A.; Cutcutache, I.; Koressaar, T.; Ye, J.; Faircloth, B.C.; Remm, M.; Rozen, S.G. Primer3new capabilities and interfaces. Nucleic Acids Res. 2012, 40, e115-e115.

10. Koressaar, T.; Remm, M. Enhancements and modifications of primer design program Primer3. Bioinformatics 2007, 23, 1289-1291, doi:10.1093/bioinformatics/btm091.

11. Pastinen, T.; Kurg, A.; Metspalu, A.; Peltonen, L.; Syvanen, A.C. Minisequencing: a specific tool for DNA analysis and diagnostics on oligonucleotide arrays. Genome Res. 1997, 7, 606-614.

12. Peakall, R.; Smouse, P.E. GenAlEx 6.5: genetic analysis in Excel. Population genetic software for teaching and research - an update. Bioinformatics 2012, 28, 2537-2539.

13. Schneider, S.; Excoffier, L.; Laval, G. Arlequin (version 3.5.1.2): An integrated software package for population genetics data analysis. Evol. Bioinform. Online 2010, 1, 47-50.

14. Rosenberg, N.A.; Li, L.M.; Ward, R.; Pritchard, J.K. Informativeness of Genetic Markers for Inference of Ancestry*. Am. J. Hum. Genet. 2003, 73, 1402-1422, doi:https://doi.org/10.1086/380416.

15. Liu, N.; Chen, L.; Wang, S.; Oh, C.; Zhao, H. Comparison of single-nucleotide polymorphisms and microsatellites in inference of population structure. BMC Genet. 2005, 6, S26, doi:10.1186/1471-2156-6-S1S26. 